\title{
Fixed drug eruption due to fluconazole
}

\author{
Jennifer Beecker MD, Sophia Colantonio BSC
}

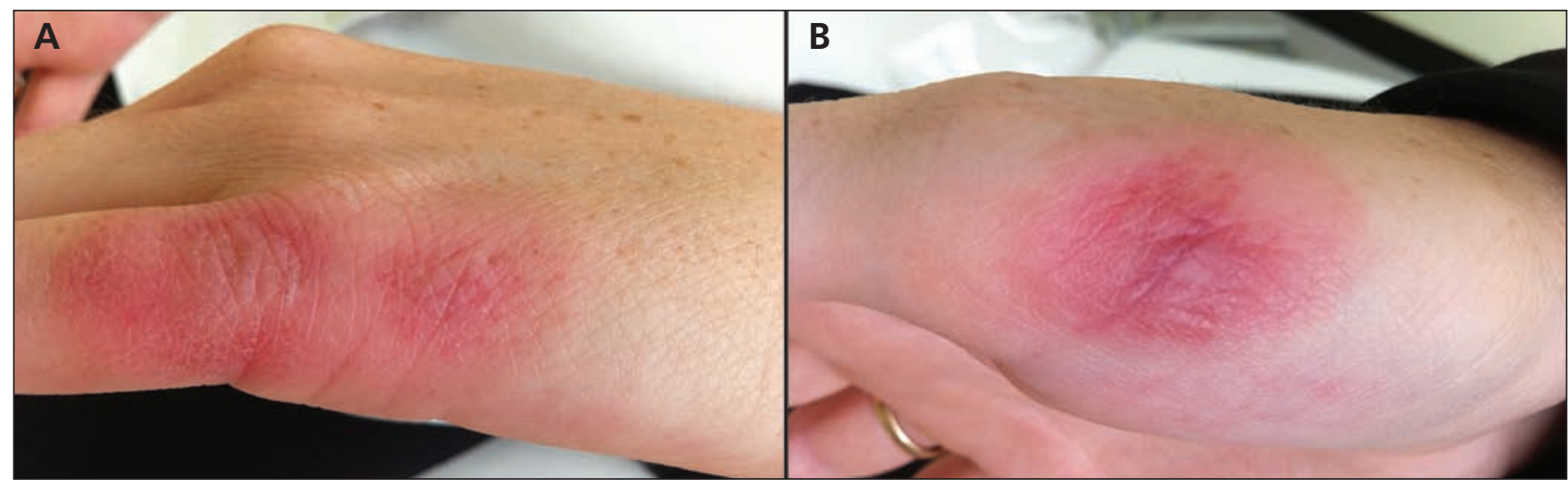

Figure 1: (A) Recurrent erythematous targetoid lesions on the hand of a 24-year-old woman with fixed drug eruption following oral administration of fluconazole. (B) Early stages of the eruption showing a single erythematous edematous plaque with a central bulla.

A healthy 24-year-old woman presented with a recurrent, raised, red rash consisting of targetoid lesions, one to two centimetres in diameter, on the backs of both hands (Figure 1A). The patient reported that the plaques became blisters (Figure 1B) and faded to circular violet patches, healing within one week with residual hyperpigmentation. The patient reported nine similar episodes in the same location, and two such episodes on her face. A typical episode began with a burning sensation, followed by itching and headache lasting 30 minutes to 24 hours. The patient thought that the eruptions might be related to the premenstrual phase of her cycle. She had no history of infection with the herpes simplex virus.

Our differential diagnosis included fixed drug eruption and erythema multiforme. Although fixed drug eruption is primarily a clinical diagnosis, we biopsied a bullous lesion and asked the patient to construct a medication diary. Histopathology of the biopsied tissue was consistent with fixed drug eruption. The patient's diary showed that the eruptions occurred after self-administered doses of oral fluconazole for recurrent vaginal candidiasis associated with the premenstrual phase of her cycle. We suggested that she discontinue the medication. Our patient had no further recurrences of the lesions and declined rechallenge. She had no reaction to the subsequent use of topical clotrimazole.
Fixed drug eruption accounts for about $11 \%-30 \%$ of all adverse drug reactions. ${ }^{1}$ Our patient had a classic fixed drug eruption. The hallmark of this reaction is the occurrence of eruptions in the same location during each episode, with additional lesions appearing upon drug rechallenge. Typical locations for the lesions include the genitals, face, hands and feet. Although fixed drug eruption is frequently confused with erythema multiforme, the histopathologies are different. Common causes of fixed drug eruption include sulphonamide drugs, tetracycline and nonsteroidal antiinflammatory drugs. Fluconazole is an uncommon cause, with only 18 published cases. ${ }^{1,2}$ Cross-reactions may occur with structurally related agents, such as itraconazole. ${ }^{3}$

Fluconazole is a commonly used antifungal agent, and fixed drug eruption should be considered a possible adverse effect of its use. Our patient's case highlights the importance of taking a comprehensive history of medication use when examining cutaneous adverse reactions.

\section{References}

1. Walling HW, Swick BL. Cutaneous fixed drug eruption to fluconazole. J Drugs Dermatol 2010;9:1025-8.

2. Tavallaee M, Rad MM. Fixed drug eruption resulting from fluconazole use: a case report. J Med Case Reports 2009;3:7368.

3. Gupta R, Thami GP. Fixed drug eruption caused by itraconazole: reactivity and cross reactivity. J Am Acad Dermtol 2008;58:521-2.
Competing interests: None declared.

This article has been peer reviewed.

Affiliations: From the Division of Dermatology (Beecker), Department of Medicine, University of Ottawa, Ottawa, Ont.; the Faculty of Medicine (Colantonio), University of Ottawa, Ottawa, Ont.; and the Yale School of Public Health (Colantonio), New Haven, Conn.

Correspondence to: Dr. Jennifer Beecker, jbeecker@rogers.com

CMAJ 2012. DOI:10.1503 /cmaj.111530 